\title{
VIII. Ueber die Gordon-Winkelmann'sche
}

Methode zur Messung von Dielectricitätsconstanten; von E. Cohn.

1. Vor einiger Zeit hat Winkelmann ${ }^{1}$ ) eine sehr einfache Methode zur Messung von Dielectricitätsconstanten (D.-C.) angegeben und ihre Brauchbarkeit an einer Reihe fester und flüssiger Substanzen geprüft. Die Resultate sind in guter Uebereinstimmung mit denjenigen, welche von anderen Beobachtern nach anderen Methoden gewonnen wurden.

Neuerdings hat nun Tschegläjew ${ }^{2}$ ) Beobachtungen angestellt nach einer Methode, welche von der Winkelmann'schen in nichts Wesentlichem abweicht. Die Resultate aber widersprechen in höchst auffälliger Weise denjenigen aller früheren Beobachter; sie ergeben nämlich durchweg die Bestätigung der Beziehung $K=n_{\infty}^{2}$, wo $K$ die D.-C. und $n_{\infty}$ den aus optischen Daten berechneten Werth des Brechungsexponenten für unendlich lange Wellen bezeichnet. Tschegläjew gibt z. B. für Wasser $K=1,75$, während alle übrigen Beobachter Werthe in der Nähe von 80 gefunden haben; um so besser stimmt freilich $\sqrt{1,75}=1,323^{3}$ ) mit $n_{\infty}=1,323$. Aehnlich verhält es sich mit Aethylalkohol und Amylalkohol.

Indem ich mir von der Entstehung dieser zweifellos falschen Versuchsresultate Rechenschaft zu geben suchte, bemerkte ich, dass der Winkelmann'schen Methode ein principieller Fehler anhaftet, dessen Einfluss wohl durch die Sorgfalt und vorurtheilsfreie Kritik des Beobachters bis zu einem gewissen Grade beseitigt werden kann, - welchen hervorzuheben ich aber um so mehr für nothwendig halte, als die Methode sich durch Einfachheit der Apparate und der Beobachtung vor allen andẹren empfiehltt.

1) Winkelmann, Wied. Ann. 38. p. 161. 1839.

2) Tschegläjew, J. d. russ. phys.-chem. Ges. 23. (2) p. 470. 1891 (russisch); Ref. Beibl. 15, p. 651.

3) Nach dem Original citirt. 
2. Winkelmann's Methode steht in engster Beziehung zu derjenigen, nach welcher bereits vor längerer Zeit Gordon ${ }^{1}$ ) D.-C. bestimmt hat. Die Veränderungen stellen sich wesentlich als Vereinfachungen dar; sie bestehen darin, dass Winkelmann erstens als Messinstrument ein Telephon statt eines Electrometers benutzt, zweitens zwei Condensatorplatten entfernt. Denken wir uns an Gordon's Versuchsanordnung die ersterwähnte Abänderung vorgenommen, die zweite aber zunächst nicht, so entsteht folgendes Schema (vgl. die Figur):

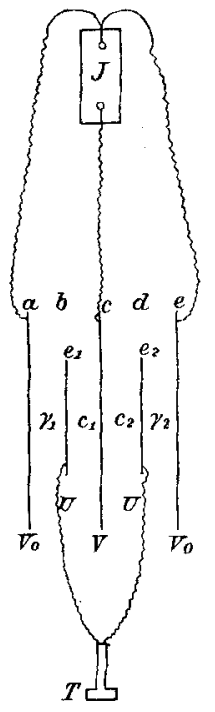

Mit dem einen Pol der secundären Rolle eines Inductoriums $I$ sind die Platten $a$ und $e$, mit dem anderen Pol ist die Platte $c$ verbunden; zwischen den Platten $b$ und $d$ befindet sich eine Leitung, in welche ein Telephon $T$ eingeschaltet ist. Ist zu beiden Seiten von $c$ alles symmetrisch, so wird das Telephon schweigen. Wird dann zwischen $a$ and $b$ eine dielectrische Schicht eingeschoben, so wird es tönen, - aber dadurch wiederum zum Schweigen gebracht werden können, dass man die Platte $a$ um eine bestimmte Strecke von $b$ entfernt. Aus der Grösse dieser Verschiebung und der Dicke der dielectrischen Schicht berechnet sich dann ihre D.-C. $K$ - wenn der Plattenabstand klein ist gegen die Dimensionen der Platten - nach dem Princip, dass eine Schicht des Dielectricums von der Dicke $d$ gleichwerthig ist einer Luftschicht von der Dicke $d / K$.

Wir haben die Bedingung für das Verstummen des Telephons analytisch zu formuliren. Die Bedingung ist diese: beim Spiel des Inductoriums müssen die Potentiale der Platten $b$ und $d$ einander gleich bleiben, ohne dass ein Austausch von Electricität zwischen ihnen stattfindet. Es seien nun die geometrischen Verhältnisse derartig, dass die Platte $b$ im elec-

1) Gordon, Treatise on Electr. and Magn. 1. p. $109 \mathrm{ff}$; auch bei Mascart, Statische Electr., deutsch von Wallentin. 1. p. $893 \mathrm{ff}$; Wiedemann, Electr. 2. p. 39. 
trischen Sinne als von $a$ und $c$ völlig eingeschlossen betrachtet werden kann (und ebenso $d$ von $c$ und $e$ ). Es bezeichne

dann ist

$$
\begin{aligned}
& V_{0} \text { das Potential von } a \text { und } e \\
& r \quad, \quad, \quad, \quad c \\
& U \quad " \quad " \quad " \quad b \text { und } d \\
& e_{1} \text { die Electricitätsmenge auf } b \\
& e_{2},, \quad, \quad, d \text {, }
\end{aligned}
$$

$$
\left\{\begin{array}{l}
e_{1}=c_{1}(U-J)+\gamma_{1}\left(U-V_{0}\right) \\
e_{2}=c_{2}(U-\dot{V})+\gamma_{2}\left(U-V_{0}\right),
\end{array}\right.
$$

wo die $c$ und $\gamma$ positive Coefficienten bezeichnen, deren Werthe sich ändern, wenn in dem betreffenden Raum (vgl. die Figur) planparallele Schichten eines anderen Dielectricums eingeschoben werden. Die gesuchte Bedingung kann also so formulirt werden: Die Coefficienten $c$ und $\gamma$ müssen solche Werthe haben, dass bei willkürlichen Aenderungen von $V$ und $V_{0}$ die Grössen $e_{1}$ und $e_{2}$ constant bleiben. I)as ergibt:

$$
\frac{c_{1}}{c_{8}}=\frac{\gamma_{1}}{\gamma_{2}}
$$

Diese Bedingungsgleichung folgt aber ebenso, wenn man nur für $V$ willkürliche Aenderungen zulässt, dagegen für $V_{0}$ einen festen Werth vorschreibt, indem man die Platten $a$ und $e$ sammt dem einen Pol des Inductoriums zur Erde ableitet.

3. Aus dieser zuletzt erwähnten Anordnung entsteht nun Winkelmann's Versuchsschema, wenn wir die Platten $a$ und $e$ in grosse Entfernung gebracht denken. ${ }^{1}$ ) I)ie Veränderung, die hierdurch hervorgebracht wird, lässt sich im allgemeinsten Falle in folgender Weise aussprechen: Bisher endeten alle Kraftlinien, die von $b$ (bez. $d$ ) ausgehen, entweder auf $a$ (bez. $e$ ) oder auf $c$. Jetzt endet ein Theil der Kraftlinien auf $c$, der Verlauf der übrigen ist durch das Versuchsschema nicht bestimmt; sie finden ihr Ende auf den Leitern der Umgebung, den Begrenzungsflächen des Zimmers, dem Körper des Beobachters. Dieser ganze von Kraftlinien erfüllte Raum bildet jetzt das Object der Messung; jede Lagenänderung von Körpern in diesem Raum ändert die Vertheilung der Kraftlinien.

1) Das Dielectricum wird dann zwischen $b$ und $c$ eingeschoben. 
Nehmen wir den einfachsten"Fall, der zugleich meistens der Wirklichkeit entsprechen wird: alle umgebenden Leiter seien - in electrostatisch ausreichender Weise - mit der Erde verbunden. Dann bleibt die Form unseres Ansatzes bestehen, nur die Werthe der Coefficienten sind geändert. Es folgt:

a) Die Coefficienten $\gamma_{1}$ und $\gamma_{2}$ werden im allgemeinen nicht einander gleich sein; das Verstummen des Telephons wird folglich im allgemeinen nicht bei symmetrischer Stellung der Platten $b$ und $d$ gegenüber $c$ (und Luft auf beiden Seiten) eintreten. So verhielt es sich thatsächlich bei Winkelmann's Versuchen. ${ }^{1}$ )

b) Dieser Umstand beeinträchtigt das Versuchsresultat nicht, sofern $\gamma_{1}$ und $\gamma_{2}$ nur während des Versuches constant bleiben, - d. h. sofern ausser der Einführung der dielectrischen Schicht im ganzen Felde keine Veränderungen vor sich gehen. Hingegen

c) jede Veränderung in der Umgebung, z. B. jeder Ortswechsel des Beobachters zwischen den beiden Theilen des Versuches, bildet eine Fehlerquelle, deren Einfluss sich der genauen Berechnung entzieht.

Wohl definirte Verhältnisse gewinnt man hier, wie bei allen electrostatischen Messungen nur dadurch, dass man das electrische Feld durch gute Leiter allseitig begrenzt. Eine Anordnung nach dem Schema unserer Figur würde dieser Forderung genügen und keinen der Vorzüge opfern, welche Winkelmann's Methode im übrigen auszeichnen.

Strassburg i. E., März 1892.

1) Winkelmann, 1. c. p. 164 . 